\title{
Phase Diagram of the Spin Hall Effect
}

\author{
E. M. Hankiewicz ${ }^{1,2, *}$ and G. Vignale ${ }^{1}$ \\ ${ }^{1}$ Department of Physics and Astronomy, University of Missouri, Columbia, Missouri 65211, USA \\ ${ }^{2}$ Department of Physics, Fordham University, Bronx, New York 10458, USA \\ (Received 30 July 2007; published 17 January 2008)
}

\begin{abstract}
We obtain analytic formulas for the frequency-dependent spin-Hall conductivity of a two-dimensional electron gas (2DEG) in the presence of impurities, linear spin-orbit Rashba interaction, and external magnetic field perpendicular to the 2DEG. We show how different mechanisms (skew scattering, side jump, and spin precession) can be brought in or out of focus by changing controllable parameters such as frequency, magnetic field, and temperature. We find, in particular, that the dc spin-Hall conductivity vanishes in the absence of a magnetic field, while a magnetic field restores the skew-scattering and side jump contributions proportionally to the ratio of magnetic and Rashba fields.
\end{abstract}

PACS numbers: 72.25.Dc, 72.25.Rb, 73.43. $-\mathrm{f}, 85.75 .-\mathrm{d}$

The spin-Hall effect (SHE), i.e., the generation of a transverse spin current in response to a dc electric field [1-6], has attracted much attention recently, particularly after a series of experiments [7-10] culminating in the observation of the SHE at room temperature [10]. On the theoretical front, however, there remains considerable uncertainty as to the physical origin of the SHE, which appears to depend on an intricate interplay of three processes: (i) the skew-scattering (SS) due to spin-orbit interaction (SOI) between electrons and impurities, (ii) the side jump (SJ) (due to the noncanonical character of the physical position and velocity variables in the presence of SOI with impurities), and (iii) the spin precession caused by spin nonconserving terms in the band structure, among which we include the linear Rashba SOI generated by an external electric field. To these we may add the influence of an external magnetic field, which tends to lock the spins in a fixed direction, thus reducing the importance of spin precession. A first principles theory that includes all of these effects on equal footing is very complicated. To our knowledge, the diagrammatic approach by Tse and Das Sarma [11] comes closest to fulfilling the order, and yet it does not include magnetic field or frequency. However, these diagrammatic calculations are very difficult to follow in detail and do not lead to an intuitive understanding of the striking nonadditive behavior of impurity and band structure effects.

Our goal in this Letter is to present the "phase diagram" of the SHE, i.e., to clarify in which range of experimentally controllable parameters one should expect the dominance of each mechanism mentioned above, and how the crossovers between different regimes occur. We do this for a 2DEG with Rashba SOI and a magnetic field perpendicular to the plane. The two parameters that are most easily controlled in an experiment are (i) the frequency $\omega$ of the electric field and (ii) Zeeman energy $\omega_{0}$. Accordingly, we plot our "phase-diagram" in the $\omega \tau-$ $\omega_{0} / \alpha_{R} k_{F}$ plane, where $\tau$ is the electron-impurity scattering time and $\alpha_{R} k_{F}$ is the magnitude of the effective mag- netic field due to Rashba SOI for electrons at the Fermi wave vector $k_{F}$. Throughout the Letter we assume $\omega, 1 / \tau$, $\alpha_{R} k_{F}, \omega_{0} \ll E_{F}$; i.e., all energy scales are smaller than the Fermi energy.

Our qualitative conclusions are shown in Fig. 1. In contrast to previous calculations [11], we found that the dc limit $(\omega \tau \rightarrow 0)$ of the spin-Hall conductivity (SHC) is zero in the presence of spin precession and in the absence of a magnetic field $\left(\omega_{0}=0\right)$. As the magnetic field increases, both SS and SJ contributions increase with the ratio of the magnetic to the Rashba field $\left(\omega_{0} / \alpha k_{F}\right)$, thus restoring the values they would have had at zero frequency and zero magnetic field in the absence of spin precession. In high-mobility samples the SS mechanism is the dominant mechanism [12,13] overcoming the SJ contribution, which has an opposite sign for attractive impurity potential. However, the SJ mechanism could well dominate in

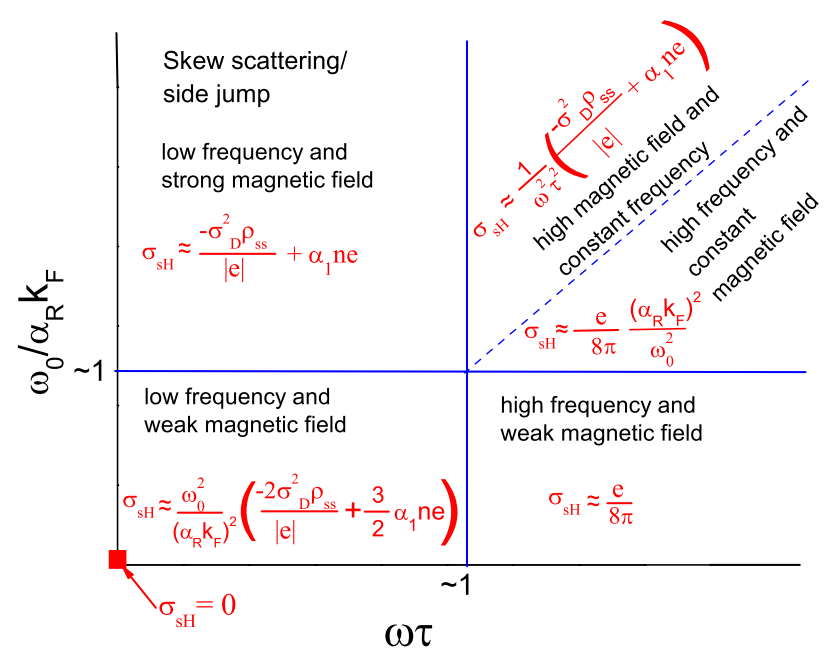

FIG. 1 (color online). Different regimes of spin-Hall effect in the $\omega \tau-\omega_{0} / \alpha_{R} k_{F}$ plane. $\sigma_{D}$ is Drude conductivity; $\rho_{\mathrm{ss}}$ is skew scattering. Spin-orbit interactions and mobility are fixed. See discussion in the text. 
low-mobility samples. As discussed in Ref. [13], the mobility can be controlled to some extent by changing the temperature $T$, and this allows one in principle to tune between SS and SJ contributions. For this reason we use the label SS/SJ for the left side of diagram, where $\omega \tau \ll 1$. In the ac regime $(\omega \tau \gg 1)$ and for low magnetic field impurities become irrelevant, leaving room for the intrinsic SHE with "universal" conductivity $e / 8 \pi$ [4]. Finally, in the ac regime and at high magnetic field the SHC declines to zero in different manners depending on whether $\omega \tau$ or the magnetic field is kept constant as shown in Fig. 1.

In what follows we describe the main points of our new theoretical approach, which enables us to calculate the spin-Hall conductivity in different regions of the parameter space and to derive simple analytic formulas describing the crossovers between different regimes.

Our model is defined by the Hamiltonian

$$
\begin{aligned}
\hat{H}= & \sum_{i=1}^{N}\left\{\frac{\hat{p}_{i}^{2}}{2 m^{*}}+V\left(\hat{\vec{r}}_{i}\right)+\frac{\alpha_{R}}{\hbar}\left(\hat{p}_{i y} \hat{S}_{i x}-\hat{p}_{i x} \hat{S}_{i y}\right)\right. \\
& \left.+\frac{\alpha_{1}}{\hbar}\left[\hat{p}_{i x} \nabla_{y} V\left(\hat{\vec{r}}_{i}\right)-\nabla_{x} V\left(\hat{\vec{r}}_{i}\right) \hat{p}_{i y}\right] \hat{S}_{i z}+\omega_{0} \hat{S}_{i z}\right\} .
\end{aligned}
$$

Besides the kinetic energy and the usual electron-impurity potential $V\left(\hat{\vec{r}}_{i}\right)$, we have two distinct spin-orbit couplings. The $\alpha_{1}$ coupling between the electrons and the impurities conserves the $z$ component of the spin and is responsible for SS and the SJ effects. The $\alpha_{R}$ coupling - also known as Rashba coupling — creates a momentum-dependent magnetic field in the plane, which breaks the conservation of $S_{z}$ and causes spin precession. This term is responsible, under appropriate conditions, for the intrinsic contribution to SHE. Finally, we have included a magnetic field perpendicular to the plane. The Zeeman splitting, $\omega_{0}$, and the frequency $\omega$ of the ac electric field (not shown in $\hat{H}$ ) are the two control knobs in terms of which our "phase diagram" will be plotted.

The skew-scattering effect is easily described in the Boltzmann equation formalism [12], but it is difficult to treat in the diagrammatic approach [11]. On the other hand, spin-precession effects are easily included in the diagrammatic formalism, but are problematic in the Boltzmann equation formalism (the distribution function becomes a $2 \times 2$ matrix). We get the best of two worlds by combining the two approaches in the following manner. First we notice that the skew-scattering collision term in the Boltzmann equation is formally equivalent to the imposition of a "spin-electric field" $E_{y}^{z}$, which accelerates upspin and down-spin electrons ("up" and "down" are defined with respect to a $z$ axis) in opposite directions along the $y$ axis, perpendicular to the flow of the charge current $(x)$. The problem is now "reduced" to calculating the $z$ spin current $j_{y}^{z}$ which flows along the $y$ axis in response to the spin-electric field $E_{y}^{z}$ in the same direction. This can be done with the help of the standard diagrammatic formalism, including both electron-impurity scattering and spin precession, but not the skew-scattering processes, for the skew scattering has already "done its job" by producing the spin-electric field $E_{y}^{z}$ (in other words, we work to first order in $\alpha_{1}$ ). Thus we have

$$
\left.j_{y}^{z}\right|_{\mathrm{ss}}=\sigma_{y y}^{z} E_{y}^{z}
$$

where $\sigma_{y y}^{z}$ is the longitudinal spin conductivity calculated in the absence of skew-scattering (or side jump) effects. On the other hand, $E_{y}^{z}$ has the well-known expression

$$
E_{y}^{z}=\rho_{\mathrm{ss}} j_{x},
$$

where $j_{x}$ is the current density in the $x$ direction and $\rho_{\text {ss }} \equiv$ $-m / n e^{2} \tau_{\mathrm{ss}}$ is the skew-scattering resistivity, calculated from the Boltzmann collision integral [12]. An explicit expression for the skew-scattering rate $1 / \tau_{\mathrm{ss}}$ is given in Eq. (29) of Ref. [12]: notice that it is proportional to $\alpha_{1}$ and positive [14]. $j_{x}$ can be expressed as $\sigma_{D} E_{x}$, where $E_{x}$ is the electric field in the $x$ direction and $\sigma_{D}$ is the Drude conductivity of the electron gas. Therefore, Eq. (2) becomes:

$$
\left.j_{y}^{z}\right|_{\mathrm{ss}}=\sigma_{y y}^{z} \rho_{\mathrm{ss}} \sigma_{D} E_{x},
$$

from which we extract the first important result of this Letter, namely, the expression for the skew-scattering contribution to the SHC $\sigma_{\mathrm{SH}}$,

$$
\sigma_{\mathrm{ss}}^{\mathrm{SH}}=\sigma_{y y}^{z} \rho_{\mathrm{ss}} \sigma_{D}
$$

The dependence of this formula on spin-precession rate, frequency of the ac field, and magnetic field will be obtained below. In particular, we will show that $\sigma_{\mathrm{ss}}^{\mathrm{SH}}$ vanishes at low magnetic field (spin-precession regime), and it recovers the zero-precession value $\left(\sigma_{D}^{2} \rho_{\text {ss }} \hbar / e\right)$ at high magnetic field. We will then consider separately side jump $\sigma_{\mathrm{sj}}^{\mathrm{SH}}$ and purely intrinsic (coming from band structure) $\sigma_{R}^{\mathrm{SH}}$ contributions to the spin-Hall conductivity.

They are most efficiently analyzed in terms of the Kubo formula for the spin-Hall conductivity. The SJ contribution, similar to SS term, vanishes at low magnetic field (spin-precession regime) and recovers its value in high magnetic fields. The remaining intrinsic contribution is easily calculated by standard diagrammatics (including vertex corrections) and leads to the well-known $e / 8 \pi$ result in the appropriate ac regime.

Skew-scattering. - As discussed above, the central role in calculating $\sigma_{\mathrm{ss}}^{\mathrm{SH}}$ is played by the longitudinal spinchannel conductivity $\sigma_{y y}^{z}$. The formal expression for the real part of $\sigma_{y y}^{z}$ is

$$
\sigma_{y y}^{z}(\omega)=-\frac{4 n e}{m^{* 2} \hbar} \frac{\Im m\left\langle\left\langle\hat{S}_{z} \hat{p}_{y} ; \hat{S}_{z} \hat{p}_{y}\right\rangle\right\rangle}{\omega},
$$

where $\hat{p}_{y}, \hat{S}_{z}$ are momentum and spin operators for a single electron [15], the double bracket is the usual notation for the spin-current-spin-current response function, and $n$ is the areal density of the electron gas. Our objective is to calculate this Kubo formula including elastic electronimpurity scattering in the Born approximation, spin precession due to a Rashba spin-orbit interaction, and a magnetic field along the $z$ axis, but neglecting spin-orbit interactions with the impurities. The Feynman diagrams 
$p_{y} s_{z}$ $p_{y} s_{z}=$
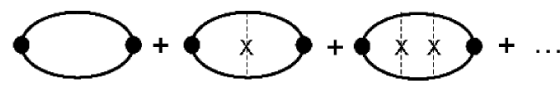

FIG. 2. Diagrammatic representation of ladder approximation to calculate the spin-channel conductivity.

for $\sigma_{y y}^{z}(\omega)$, to the desired level of accuracy, are shown in Fig. 2. The solid lines represent $2 \times 2$ Green's functions including Rashba spin-orbit coupling, magnetic field, and a diagonal disorder self-energy $(i / 2 \tau) \operatorname{sgn}(\omega)$, where $1 / \tau$ is the elastic scattering rate:

$$
\hat{G}(p, \omega)=\frac{\omega-\epsilon_{p}+\vec{h}_{p} \hat{\vec{S}}+\frac{i}{2 \tau} \operatorname{sgn}(\omega)}{\left[\omega-\epsilon_{p}+\frac{i}{2 \tau} \operatorname{sgn}(\omega)\right]^{2}-h_{p}^{2} / 4} .
$$

Here $\vec{h}_{p}=\left(\alpha_{R} k_{y},-\alpha_{R} k_{x}, \omega_{0}\right)$ combines the Rashba inplane field ( $x$ and $y$ components) and the magnetic field along the $z$ axis in a single effective field. The dashed lines are electron-impurity interactions (averaged over disorder), and there are spin-current operators $p_{y} S_{z}$ at the vertices. The empty bubble, calculated by the standard procedure with $\alpha_{R} k_{F}, \omega_{0}$, and $1 / \tau$ all much smaller than the Fermi energy, is

$$
\left.\sigma_{y y}^{z}(\omega)\right|_{\text {bubble }} \simeq \frac{\hbar}{e} \frac{\sigma_{D}}{1+(\omega \tau)^{2}} \frac{1+\omega_{0}^{2} \tau^{2}}{1+\Omega^{2} \tau^{2}},
$$

where $\sigma_{D}=n e^{2} \tau / m^{*}$ is the Drude conductivity and $\Omega=$ $\sqrt{\left(\alpha_{R} k_{F}\right)^{2}+\omega_{0}^{2}}$ is the spin-precession frequency. Equation (8) suggests that the spin-channel conductivity is similar to the ordinary Drude conductivity, only somewhat reduced by precession about the in-plane Rashba field. The inclusion of vertex corrections changes the result drastically. The sum of the ladder diagrams produces a "dressed" spin-current vertex $\hat{\Lambda}_{y}^{z}$ of the form:

$$
\hat{\Lambda}_{y}^{z}=\hat{p}_{y} \hat{S}_{z}-\frac{\alpha_{R} k_{F}^{2}\left[\left(\omega_{0} \tau\right) \hat{S}_{x}-(1-i \omega \tau) \hat{S}_{y}\right]}{\alpha_{R}^{2} k_{F}^{2} \tau-2 \Omega^{2} \tau(1-i \omega \tau)} .
$$

Including $\hat{\Lambda}_{y}^{z}$ in the calculation of the spin-channel conductivity we get

$$
\sigma_{y y}^{z}(\omega)=\frac{2 \hbar \sigma_{D}}{e} \frac{\cos ^{2} \delta\left(1+\cos ^{2} \delta\right)}{\left(1+\cos ^{2} \delta\right)^{2}+4 \omega^{2} \tau^{2}}
$$

where $\cos \delta=\frac{\omega_{0}}{\Omega}$ is the cosine of the angle between the external magnetic field and the full effective magnetic field $\vec{\Omega}$. While Eq. (10) reduces to the empty-bubble result in the limit $\omega_{0} \gg \alpha_{R} k_{F}$, its most striking feature is that it vanishes identically (i.e., at all frequencies) in the opposite limit $\omega_{0} \ll \alpha_{R} k_{F}$. In other words, the spin-channel conductivity is zero in the absence of an external magnetic field, as long as an infinitesimal spin-precession rate $\alpha_{R} k_{F}$ is present. Accordingly, the skew-scattering spin-Hall conductivity from Eq. (5) is [16]

$$
\sigma_{\mathrm{ss}}^{\mathrm{SH}}=\frac{2 \hbar \sigma_{D}^{2} \rho_{\mathrm{ss}}}{e} \frac{\cos ^{2} \delta\left(1+\cos ^{2} \delta\right)}{\left(1+\cos ^{2} \delta\right)^{2}+4 \omega^{2} \tau^{2}}
$$

which vanishes in the absence of an external magnetic field and recovers the value $\sigma_{D}^{2} \rho_{\mathrm{ss}} \hbar / e$ for strong magnetic fields. The vanishing of the spin conductivity is a peculiar feature of the linear Rashba model, in which the spin current $\hat{p}_{y} \hat{S}_{z}$ is proportional to the time derivative of $\hat{S}_{y}$ : $\hat{p}_{y} \hat{S}_{z}=-\hat{S}_{y} / \alpha_{R}$. The expectation value of a time derivative must vanish at zero frequency. This is the same reason which causes the vanishing of the Rashba spin-Hall conductivity in the do limit.

Side jump. - In a recent Letter [13] we have shown how to identify the SJ contribution in the Kubo linear response formalism. We perturb the Hamiltonian (1) with a uniform electric field of frequency $\omega$ in the $x$ direction. After a series of manipulations, which have been described in Ref. [13], we arrive at the following Kubo formula for SHC:

$$
\begin{aligned}
\sigma_{y x}^{\mathrm{SH}}(\omega)= & \frac{n e}{i m^{*} \omega}\left(\left\langle\left\langle\hat{p}_{y} \hat{S}_{z} ; \frac{\hat{p}_{x}}{m^{*}}-\frac{\alpha_{R}}{\hbar} \hat{S}_{y}\right\rangle\right\rangle\right) \\
& +\frac{\alpha_{1} n e}{2 i m^{*} \omega}\left(\frac{4\left\langle\left\langle\hat{p}_{y} \hat{S}_{z} ; \nabla_{y} V(\hat{\vec{r}}) \hat{S}_{z}\right\rangle\right\rangle}{\hbar^{2}}-\left\langle\left\langle\nabla_{x} V(\hat{\vec{r}}) ; \hat{p}_{x}\right\rangle\right\rangle\right) .
\end{aligned}
$$

The first term on the right-hand side produces SS as well as possible "intrinsic" contributions which are discussed in next section. The second line of this equation, which explicitly shows a dependence on $\alpha_{1}$, is responsible for the $\mathrm{SJ}$ contribution [17], denoted $\sigma_{\mathrm{sj}}^{\mathrm{SH}}$. We now focus on this contribution. Using the Heisenberg equation of motion for the momentum operator $\dot{\hat{p}}_{i x}=-\nabla_{x} V\left(\hat{\vec{r}}_{i}\right)$ in zero order in $\alpha_{1}$ (because SJ terms are already explicitly linear in $\alpha_{1}$ ), we rewrite the SJ contribution as

$$
\sigma_{\mathrm{sj}}^{\mathrm{SH}}(\omega)=\frac{e \alpha_{1} n}{2 i m^{*} \omega}\left[\left\langle\left\langle\dot{\hat{p}}_{x} ; \hat{p}_{x}\right\rangle\right\rangle-\frac{4\left\langle\left\langle\hat{p}_{y} \hat{S}_{z} ; \dot{\hat{p}}_{y} \hat{S}_{z}\right\rangle\right\rangle}{\hbar^{2}}\right] .
$$

In the first term in the square brackets we apply the standard rule of integration, which allows us to replace $\dot{\hat{p}}_{x} / i \omega$ by $-\hat{p}_{x}$ and rewrite it (its real part) as:

$$
\frac{\alpha_{1} e n}{2 i m^{*} \omega}\left\langle\left\langle\dot{\hat{p}}_{x} ; \hat{p}_{x}\right\rangle\right\rangle=\frac{-\alpha_{1} n e}{2 m^{*}}\left\langle\left\langle\hat{p}_{x}, \hat{p}_{x}\right\rangle\right\rangle=\frac{\alpha_{1} n e}{2\left(1+\omega^{2} \tau^{2}\right)},
$$

where the last equality follows from the well-known form of the current-current response function in a weakly disordered system. The second term in the square brackets of Eq. (13) can be rewritten as follows:

$-\frac{1}{i \omega}\left\langle\left\langle\hat{p}_{y} \hat{S}_{z} ; \dot{\hat{p}}_{y} \hat{S}_{z}\right\rangle\right\rangle=-\frac{1}{i \omega}\left\langle\left\langle\hat{p}_{y} \hat{S}_{z} ; \frac{d}{d t}\left(\hat{p}_{y} \hat{S}_{z}\right)-\hat{p}_{y} \dot{\hat{S}}_{z}\right\rangle\right\rangle$.

Applying again the integration formula, the first term of Eq. (15) yields [18]:

$$
\frac{-2 e n \alpha_{1}}{m^{*}}\left\langle\left\langle\hat{p}_{y} \hat{S}_{z} ; \hat{p}_{y} \hat{S}_{z}\right\rangle\right\rangle=\frac{e \alpha_{1} n}{2} \frac{\cos ^{2} \delta\left(1+\cos ^{2} \delta\right)^{2}}{\left(1+\cos ^{2} \delta\right)^{2}+4 \omega^{2} \tau^{2}} .
$$

In the absence of spin precession (i.e., for $\omega_{0} \gg \alpha_{R} k_{F}$, $\cos \delta \simeq 1$ ) and for zero frequency the contributions (14) and (16) add up to the "usual" SJ conductivity $\alpha_{1}$ ne of 


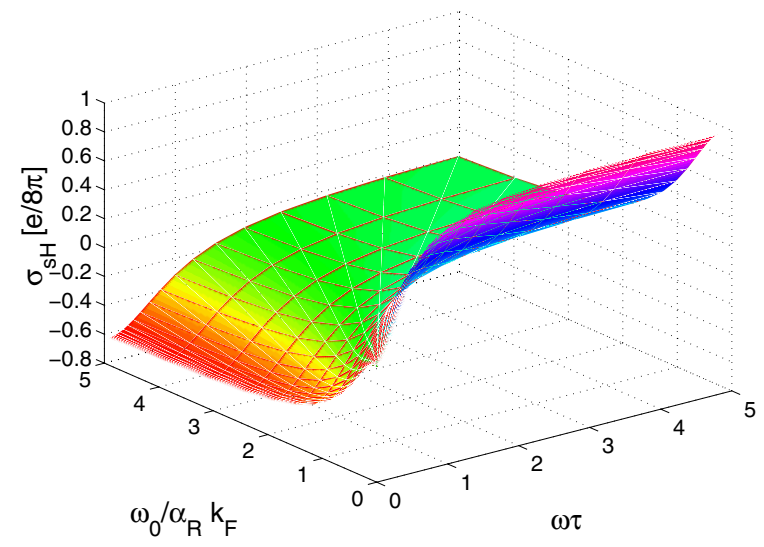

FIG. 3 (color online). Behavior of spin-Hall conductivity as a function of $\omega \tau$ and $\omega_{0} / \alpha_{R} k_{F}$ and carrier concentration $n_{2 D}=2 \times 10^{12} \mathrm{~cm}^{-2}, \quad \mu=1 \mathrm{~m}^{2} / \mathrm{V} \mathrm{s}, \quad m=0.067 m_{e}, \quad \alpha_{1}=$ $0.053 \mathrm{~nm}^{2}, \alpha_{R} k_{F}=10 \mathrm{meV}$. For calculation of skew scattering, we assumed square potential characterized by $\tau / \tau_{\mathrm{ss}}=0.002$ like in Ref. [12].

Ref. [13]. In the opposite limit of $\omega_{0} \ll \alpha_{R} k_{F}(\cos \delta=0)$ this term vanishes for the same reason as $\sigma_{\mathrm{ss}}^{\mathrm{SH}}$. The second term on the right-hand side of Eq. (15) can be rewritten as $\left\langle\left\langle\hat{S}_{y} ; \hat{p}_{y}(\hat{\vec{p}} \cdot \hat{\vec{S}})\right\rangle\right\rangle$ (we use the equations of motion $\dot{\hat{S}}_{z}=$ $\alpha_{R} \hat{\vec{p}} \cdot \hat{\vec{S}}$ and $\left.\hat{p}_{y} \hat{S}_{z}=-\dot{\hat{S}}_{y} / \alpha_{R}\right)$. In the limit of zero frequency and zero magnetic field this reduces to $\left\langle\left\langle\hat{p}_{y} \hat{S}_{y} ; \hat{p}_{y} \hat{S}_{y}\right\rangle\right\rangle=\left(\hbar^{2} / 4\right)\left\langle\left\langle\hat{p}_{x} ; \hat{p}_{x}\right\rangle\right\rangle$, which exactly cancels the contribution from (14) and causes the SJ contribution to vanish. For general magnetic fields and frequencies the total $\sigma_{\mathrm{sj}}^{\mathrm{SH}}$ is finally given by the formula [19]

$$
\sigma_{\mathrm{sj}}^{\mathrm{SH}}(\omega)=\frac{\alpha_{1} n e}{2}\left[\frac{\cos ^{2} \delta}{1+\omega^{2} \tau^{2}}+\frac{2 \cos ^{2} \delta\left(1+\cos ^{2} \delta\right)}{\left(1+\cos ^{2} \delta\right)^{2}+4 \omega^{2} \tau^{2}}\right],
$$

which in the dc limit simplifies to

$$
\sigma_{\mathrm{sj}}^{\mathrm{SH}}(\omega)=\frac{\alpha_{1} n e \cos ^{2} \delta}{2}\left(\frac{3+\cos ^{2} \delta}{1+\cos ^{2} \delta}\right) .
$$

Summarizing, the side jump conductivity in the dc limit $(\omega \tau \ll 1)$ grows from zero at $\omega_{0}=0$ to the "full" value $\left(\alpha_{1} n e\right)$ at high magnetic fields, while it tends to zero in high frequency limit.

Rashba contribution. -Evaluating the first line of Eq. (12) in the absence of skew scattering and side jump, which we have already taken into account, leads to the well-known result $[20,21] \sigma_{R}^{\mathrm{SH}}(\omega)=\frac{e}{8 \pi} \frac{(\omega \tau)^{2}}{(\omega \tau)^{2}+1 / 4}$, which vanishes at $\omega=0$ and tends to the "ballistic" limit $e / 8 \pi$ for $\omega \gg 1 / \tau$. We have found that the behavior of $\sigma_{R}^{\mathrm{SH}}(\omega)$ as a function of external perpendicular magnetic field and frequency is given by:

$$
\sigma_{R}^{\mathrm{SH}}(\omega)=\frac{e}{8 \pi} \frac{\alpha_{R}^{2} k_{F}^{2}}{\Omega^{2}} \frac{4 \omega^{2} \tau^{2}}{\left(1+\cos ^{2} \delta\right)^{2}+4 \omega^{2} \tau^{2}},
$$

which is a decreasing function of magnetic field.
Conclusions. - The results of our analysis are summarized in Fig. 3 which shows the full spin-Hall conductivity $\sigma_{\mathrm{SH}}$, including Rashba, skew-scattering, and side jump terms, as a function of two variables, frequency and magnetic field, for realistic values of the parameters. The figure shows the smooth crossovers between different regimes and should be useful to experimentalists attempting to extricate the various components of this still quite intriguing effect.

We thank H. A. Engel for useful discussions. This work was supported by NSF Grant No. DMR-0313681.

*hankiewicz@fordham.edu

[1] M. I. Dyakonov and V.I. Perel, Zh. Eksp. Teor. Fiz. 13, 657 (1971) [JETP Lett. 13, 467 (1971)].

[2] J.E. Hirsch, Phys. Rev. Lett. 83, 1834 (1999).

[3] S. Zhang, Phys. Rev. Lett. 85, 393 (2000).

[4] J. Sinova et al., Phys. Rev. Lett. 92, 126603 (2004).

[5] S. Murakami, N. Nagaosa, and S.-C. Zhang, Science 301, 1348 (2003).

[6] R. Raimondi and P. Schwab, Phys. Rev. B 71, 033311 (2005).

[7] Y. K. Kato et al., Science 306, 1910 (2004).

[8] V. Sih et al., Nature Phys. 1, 31 (2005).

[9] J. Wunderlich et al., Phys. Rev. Lett. 94, 047204 (2005).

[10] N. P. Stern et al., Phys. Rev. Lett. 97, 126603 (2006).

[11] W. K. Tse and S.D. Sarma, Phys. Rev. B 74, 245309 (2006).

[12] E. M. Hankiewicz and G. Vignale, Phys. Rev. B 73, 115339 (2006).

[13] E. M. Hankiewicz, G. Vignale, and M. Flatté, Phys. Rev. Lett. 97, 266601 (2006).

[14] Spin-precession corrections to Eq. (3) are of order of $\alpha_{R}^{2} \alpha_{1}$ and we omit them as small.

[15] Substitution of operator for total system $\sum_{i=1}^{N} \hat{p}_{i y} S_{i z}$ by sum of one-electron operators $N p_{y} S_{z}$ is justified because we omit electron-electron interactions.

[16] Actually, this is not the complete formula for the skewscattering contribution (which is quite complicated), but a simplified version of it, which is valid in the limit of $\alpha_{R} k_{F} \tau \gg 1$. However, even in the opposite regime $\alpha_{R} k_{F} \tau \ll 1$ this formula remains qualitatively correct, in the sense that it exhibits the correct scaling behaviors in the limits of weak and high magnetic field, low and high frequency. Notice that contributions of order $\omega^{2} / \Omega^{2}$, which we have omitted, give finite SS at finite frequency and zero magnetic field, but are negligible in comparison to the larger intrinsic contribution.

[17] We omitted corrections of order $\alpha_{R}^{2} \alpha_{1}$.

[18] Equation (16) can be found from Eq. (10) using KramersKrönig relation.

[19] Again, we present only a simplified version of the complete formula, subject to restrictions explained in Ref. [16].

[20] J. I. Inoue, G. E. W. Bauer, and L. W. Molenkamp, Phys. Rev. B 70, 041303(R) (2004).

[21] E. G. Mishchenko, A. V. Shytov, and B. I. Halperin, Phys. Rev. Lett. 93, 226602 (2004). 